\title{
Drug-Resistant Tuberculosis: Correlation between Positivity of Acid-Fast Bacilli Sputum and Time to Conversion on Patients with Short-Term Treatment Regimen
}

\author{
Tutik Kusmiati* (D), Anita Nur Charisma ${ }^{(D)}$, Nur Prasetyo Nugroho $\mathbb{D}$ \\ and Soedarsono
}

Department of Pulmonology and Respiratory Medicine, Faculty of Medicine, Universitas Airlangga - Dr. Soetomo General Academic Hospital, Surabaya, Indonesia.

\begin{abstract}
Drug-resistant tuberculosis (DR-TB) is a worldwide global burden and related to poor treatment outcomes. Monitoring the progress of DR-TB treatment can be carried out microscopic with Acid-Fast Bacilli (AFB) sputum smear and assessed from the beginning or the first time the patient is diagnosed and monthly to determine the sequential conversion of AFB baseline to the next month twice until it becomes negative. The prolonged conversion has been associated with infectiousness and treatment outcomes. This study was aimed to determine the correlation between positivity of AFB sputum and time to conversion in DR-TB patients with short-term treatment (STR) regimen. An analytic retrospective at hospital collected from medical records of DR-TB patients, from September 2017 to July 2018. Spearman technique was used to analyze the data with $p<0.05$. From the total of 151 DR-TB patients on STR regimen, 51 patients were enrolled consisting of $30(58.8 \%)$ males and $21(41.2 \%)$ females with ages average were $51 \pm 12.9$ years old. Overall, 39 (76.5\%) patients had time of AFB conversion in the first month, $9(17.6 \%)$ patients in the second months, $2(3.9 \%)$ patients in the third months, and $1(2 \%)$ patient in the fourth months. Among those patients, $26(51 \%)$ patients had completed the treatment outcomes, 22 (43.1\%) were loss to follow-up, 1 (2\%) patient had the treatment failure, and 2 (3.9\%) were died. There was no significant correlation between AFB sputum baseline (Scanty, 1+, 2+, 3+) with the time to sputum conversion $(p>0.05)$. AFB sputum have significant correlation with time of culture conversion $(p<0.05)$, and treatment compliance was not associated with time of AFB sputum conversion $(p>0.05)$. There was no significant relation between positivity of AFB baseline and time to sputum conversion in DR-TB patients on short-term treatment (STR) regimen. AFB sputum have significant correlation with time of culture conversion in DR-TB patients on short-term treatment (STR) regimen.
\end{abstract}

Keywords: Time to conversion, sputum smear, drug-resistant tuberculosis

*Correspondence: tut.kusmiati@gmail.com

(Received: February 10, 2020; accepted: October 17, 2020)

Abbreviations: DR-TB (Drug-Resistant Tuberculosis), RR / MDR, (Rifampisin Resistant / Multi-drug Resistant), AFB (Acid-Fast Bacilli), WHO (World Health Organization), STR (Short-term treatment).

Citation: Kusmiati T, Charisma AN, Nugroho NP, Soedarsono. Drug-Resistant Tuberculosis: Correlation between Positivity of AcidFast Bacilli Sputum and Time to Conversion on Patients With Short-Term Treatment Regimen. J Pure Appl Microbiol. 2020;14(4): 2443-2451. doi: 10.22207/JPAM.14.4.22

(C) The Author(s) 2020. Open Access. This article is distributed under the terms of the Creative Commons Attribution 4.0 International License which permits unrestricted use, sharing, distribution, and reproduction in any medium, provided you give appropriate credit to the original author(s) and the source, provide a link to the Creative Commons license, and indicate if changes were made. 


\section{INTRODUCTION}

The World Health Organization (WHO) ranked Tuberculosis (TB) $10^{\text {th }}$ in the world and leading cause of death in single infectious agents. The incidence of DR-TB between 2016 and 2017, increased by more than $30 \%$ in six of the 30 countries that had the highest burden of DR-TB (Angola, Korea's Democratic Republic of Korea, Indonesia, Nigeria, Somalia and Thailand). Globally, the number of DR-TB cases (RR/MDR) has increased, in 2017 was $29 \%$ of the estimated 558.000 case incidents and $49 \%$ of the estimated 330.000 cases of DR-TB (RR/MDR) were notified and the treatment success remains low ${ }^{1}$.

Based on the Global Tuberculosis Report 2018 (WHO), in Indonesia was reported burden of DR-TB (RR/MDR) estimated incidence has a value of $23,000(16,000-57,000)$ with a calculation of 8.8 (6.2-12 per 100.000 population), with estimates of DR-TB (RR/MDR) cases of 12,000 $(8,600-15,000)$ or as many as $13 \%(9-18)$ of the new $2.4 \%$ cases (1.8-3.3). While the success rate of RR/MDR TB treatment in 2017 was 55\%. Therefore previous DR-TB treatment with conventional DR-TB regimen was still considered to require a long time and could affect the low success rate of treatment ${ }^{1}$. WHO released guidelines for the management of DR-TB with short-term treatment (STR) regimen of 9-12 months to update the existing conventional regimen and that can be given to certain DR-TB conditions or cases in order to reduce treatment failure ${ }^{2,3}$. In 2011, the previous guidelines recommended in an intensive treatment phase duration of 8 months and 20 months of total treatment ${ }^{4,5}$. Monitoring the progress of DR-TB treatment can be carried out microscopic with Acid-Fast Bacilli (AFB) sputum smear and assessed from the beginning or the first time the patient is diagnosed and monthly to determine the sequential conversion of AFB baseline to the next month twice until it becomes negative. The prolonged conversion has been associated with infectiousness, and monitoring serial sputum and culture important for clinical management, therapeutic planning and related to treatment outcomes ${ }^{2,6}$. Based on that, it is considered to be a concern and needs to know the correlation between positivity of AFB sputum and time to conversion in DR-TB patients on STR regimen ${ }^{2,7}$. This study was aim to determined the correlation between positivity of AFB sputum and time to conversion in DR-TB patients with STR regimen.

\section{MATERIALS AND METHODS Study design and settings}

This study used a retrospective design. Data collected from medical records of DR-TB (RR/MDR) patients at the Dr. Soetomo General Academic Hospital, Surabaya, Indonesia. The population was 151 DR-TB patients on STR regimen from September 2017 to July 2018, 51 patients were enrolled using purposive sampling technique and according to inclusion and exclusion selection criteria.

\section{Participant}

The inclusion criteria in this study were: DR-TB patients who received STR regimen and fulfilled the treatment criteria, patients who performed AFB sputum examination and Gene Xpert ${ }^{\circledR}$ MTB/RIF showed RR results. The exclusion criteria were patients who died before the AFB sputum results occur in conversion, patients who cannot expel sputum and patients loss to followup before the conversion.

\section{Design}

Referred to WHO, positivity (number) of AFB was defined as the number of bacilli present in patient's sputum is in direct relation to degree of infectiousness. Method for smears stained by Ziehl-Neelsen/acid fast bacilli stain (immersion lens $\times 100$ ). This staining allows the detection of the acid fast microorganisms, mainly Mycobacteria spp. The most infectious TB patients can be detected rapidly using direct microscopy examination, sensitivity of the sample examined contain at least 10.000 bacilli (per $\mathrm{ml}$ ) in order to be seen positive on microscopy. High number of bacilli found in patients with cavitary pulmonary TB since the cavity formation will increase oxygen concentration, favor the bacillary growth and also creating an environment that prevent immunological control ${ }^{8,9}$ (Table 1).

Time to AFB sputum and culture conversion, was the interval from the beginning or the first time the patients diagnosed and monthly to determine the sequential conversion of baseline positive to the next month twice until it becomes negative. Completed treatment outcome according to defined treatment duration. 
Patients who stops treatment for two consecutive months or more, assessed as loss to follow-up. Time to conversion more than six months or obtained second-line drug-resistance considered of outcomes treatment failure. Died; A patient who dies for any reason during treatment, in this study was defined patients who died after conversion ${ }^{2}$. Patients compliance in this study was defined the rate of patients to carry out the treatment that has been determined in; <2 weeks (good compliance), $>2$ weeks - 1 month (moderate compliance,) 1 month - 2 months (poor compliance), and $>2$ months (non-compliance).

Fulfilled treatment criteria DR-TB patients who received STR regimen, base on Ministry of Health of the Republic of Indonesia was classified: no evidence of fluoroquinolone resistance or second-line drugs injection, no contact with Pre-XDR/XDR-TB patients, never get second-line treatment for more than one month, no STR regimen intolerance, no pregnancy, not extrapulmonary cases, there is no risk of an unfavorable outcome ${ }^{2}$.

Table 1. Positivity of AFB sputum

\begin{tabular}{ll}
\hline Number of AFB & Grade of positivity \\
\hline Scanty & 1-9 AFB per 100 immersion fields \\
$1+$ & $10-99$ AFB per 100 immersion fields \\
$2+$ & $1-10$ AFB per field \\
$3+$ & $\geq 10$ AFB per field
\end{tabular}

\section{Statistical analysis}

Data were coded, entered, cleaned and analyzed using the statistical software SPSS for Windows version 20.0 (IBM Corporation, New York, USA) and Microsoft Excel 2010 Software. Frequencies and proportions were used to describe demographic patients and clinical characteristics. Demographic data included age, sex, and diagnose of DR-TB. Clinical and microbiological data included AFB baseline sputum, time to AFB conversion, time to culture conversion, treatment compliance and outcomes. The model included the following variables: AFB baseline sputum and compliance as independent variables, and the time to sputum and culture conversion as dependent variables.

The correlation between AFB sputum and time to AFB conversion, culture conversion and treatment compliance were analyzed using Spearman. Proportions were computed for categorical variables and expressed as percentages, means, and standard deviation (SD). P value ( $p<$ 0.05 ) was considered statistically significant.

\section{RESULTS}

\section{Demographic and clinical characteristic}

Total of 151 DR-TB patients on STR regimen with GeneXpert ${ }^{\circledR}$ MTB/RIF showed RR results. 100 patients were excluded and did not meet inclusion criteria, following of $(n=62)$ loss to follow-up and/or $(n=4)$ died before conversion

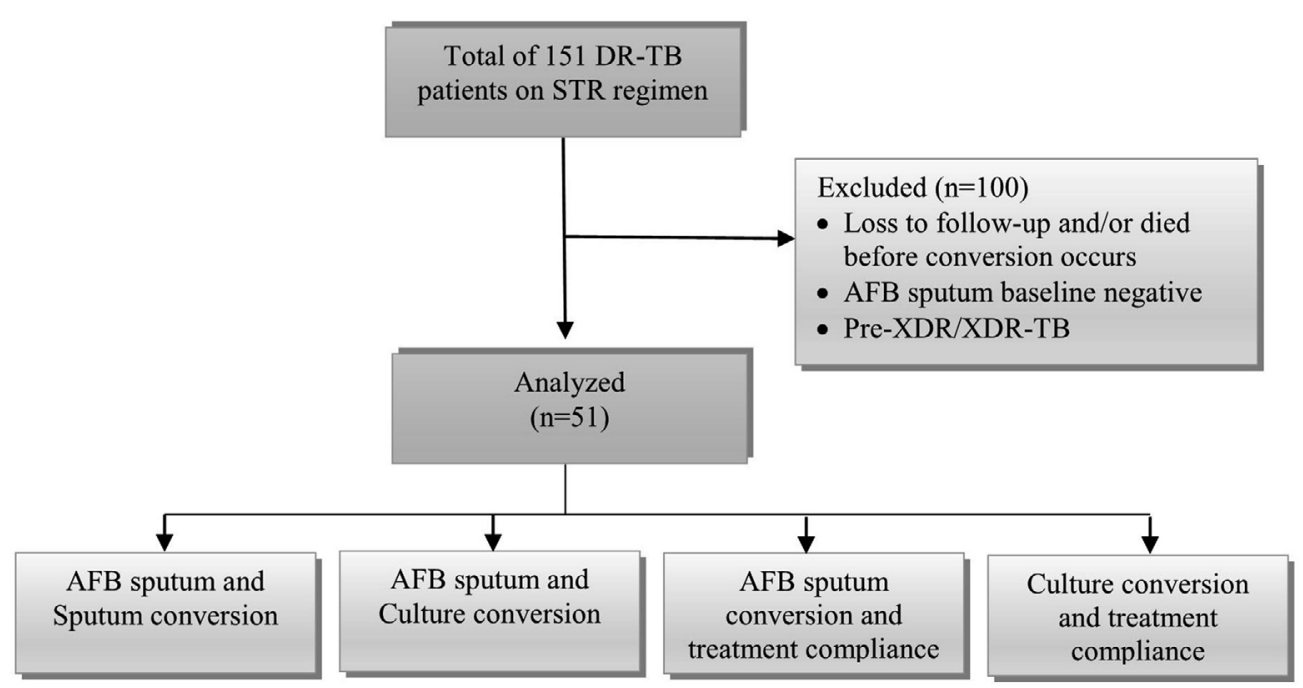

Fig. 1. Study flow diagram. A total 51 DR-TB patients on STR regimen comprised the study population after excluding patients. 
occurs, $(n=27)$ patients showed AFB sputum baseline negative, $(n=7)$ was diagnosed Pre-XDR/ XDR-TB (Fig. 1). The remaining 51 patients were included in the analysis as samples, a majority of patients mean age was $51 \pm 12.9$ years old. Patients were predominantly $30(58.8 \%)$ males and 21 (41.2\%) females (Table 2 ).

Table 2. General demographic and clinical characteristics

\begin{tabular}{|c|c|}
\hline Variable & $\mathrm{n}(\%)$ \\
\hline \multicolumn{2}{|l|}{ Range age } \\
\hline$<30$ years & $7(13.7)$ \\
\hline $30-40$ years & $10(19.6)$ \\
\hline $41-50$ years & 15 (19.4) \\
\hline $51-60$ years & $13(25.5)$ \\
\hline$>60$ years & $6(11.8)$ \\
\hline \multicolumn{2}{|l|}{ Sex } \\
\hline Male & $30(58.8)$ \\
\hline Female & $21(41.2)$ \\
\hline \multicolumn{2}{|l|}{ Diagnose of DR-TB } \\
\hline RR-TB & $17(33.3)$ \\
\hline MDR-TB & $34(66.7)$ \\
\hline \multicolumn{2}{|l|}{ AFB Baseline sputum } \\
\hline Scanty & $9(17.6)$ \\
\hline $1+$ & $13(25.5)$ \\
\hline $2+$ & $13(25.5)$ \\
\hline $3+$ & $16(31.4)$ \\
\hline \multicolumn{2}{|c|}{ AFB sputum conversion (time) } \\
\hline $1^{\text {st }}$ month & 39 (76.5) \\
\hline $2^{\text {nd }}$ months & $9(17.6)$ \\
\hline $3^{\text {rd }}$ months & $2(3.9)$ \\
\hline $4^{\text {th }}$ months & $1(2.0)$ \\
\hline $5^{\text {th }}$ months & $0(0.0)$ \\
\hline$\geq 6^{\text {th }}$ months & $0(0.0)$ \\
\hline \multicolumn{2}{|c|}{ Culture conversion (time) } \\
\hline $1^{\text {st }}$ month & $22(43.1)$ \\
\hline $2^{\text {nd }}$ months & $25(49.0)$ \\
\hline $3^{\text {rd }}$ months & $3(5.9)$ \\
\hline $4^{\text {th }}$ months & $0(0.0)$ \\
\hline $5^{\text {th }}$ months & $1(2.0)$ \\
\hline$\geq 6^{\text {th }}$ months & $0(0.0)$ \\
\hline \multicolumn{2}{|c|}{ Treatment compliance (time) } \\
\hline$<2$ weeks & $7(13.7)$ \\
\hline \multicolumn{2}{|l|}{2 weeks - 1 month } \\
\hline 1 month -2 months & $9(17.6)$ \\
\hline \multicolumn{2}{|l|}{$10(19.7)$} \\
\hline$>2$ months & $25(49.0)$ \\
\hline \multicolumn{2}{|l|}{ Outcomes } \\
\hline Completed & $26(51.0)$ \\
\hline Loss to follow up & $22(43.1)$ \\
\hline Treatment failure & $1(2.0)$ \\
\hline Died & $2(3.9)$ \\
\hline
\end{tabular}

Following of 17 (33.3) RR-TB patients and 34 (66.7) MDR-TB patients with AFB baseline positive, among 9 (17.6\%) was showed Scanty, 13 (25.5\%) showed $1+, 13(25.5 \%)$ showed $2+$ and 16 (31.4\%) showed 3+. Overall, 39 (76.5\%) patients had time of AFB conversion in the first month, 9 (17.6\%) patients in the second months, 2 (3.9\%) patients in the third months, and $1(2 \%)$ patient in the fourth months.

Time of treatment compliance from DRTB patients on STR regimen in hospital represented $7(13.7 \%)$ patients compliance less than two weeks and with the most comparison $25(49 \%)$ treatment compliance more than two months. Among those patients, $26(51 \%)$ patients had completed the treatment outcomes, 22 (43.1\%) were loss to follow-up, 1 (2\%) patient had the treatment failure, and 2 (3.9\%) were died.

\section{AFB sputum and sputum conversion}

The time of AFB sputum conversion can be seen in $9(100 \%)$ patients with AFB baseline Scanty in the first month. Patients with AFB baseline $1+(n=13)$ respectively showed $9(69.2 \%)$ conversion in the first month, 4 (30.8\%) in the second months. Patients with AFB baseline $2+$ ( $n$ $=13$ ) respectively showed $8(6.15 \%)$ conversion in the first month, $2(15.4 \%)$ patients in the second months, $2(15.4 \%)$ in the third months and 1 (7.7\%) patient in the fourth months. Patients with AFB baseline $3+(n=16)$ respectively showed $13(81.8 \%)$ conversion in the first month dan 3 $(18.8 \%)$ patients in the second months. There was no significant correlation between AFB sputum baseline (Scanty, $1+, 2+, 3+$ ) with the time to AFB sputum conversion ( $p=0.528$; table 3 ).

\section{AFB Sputum and culture conversion}

Overall, $22(43.1 \%)$ patients had time of culture conversion in the first month, 25 (49\%) patients in the second months, 3 (5.9\%) patients in the third months, there is no culture conversion $(0 \%)$ in the fourth months, and $1(2 \%)$ patient had time of culture conversion in the fifth months (Table 2).

Time to culture conversion with AFB baseline sputum correlation showed 7 (77.8\%) patients with AFB baseline Scanty was conversion in first month and $2(22.2 \%)$ in second months. Patients with AFB baseline $1+(n=9)$ respectively showed conversion $6(46.2 \%)$ in first month, 6 $(46.2 \%)$ in second months and $1(77.7 \%)$ in third 
months. Patients with AFB baseline $2+(n=13)$ respectively showed 4 (30.8\%) in first month, 7 (53.8\%) patients in second months, 1 (7.7\%) patient in third months and $1(7.7 \%)$ patient in fifth months. Patients with AFB baseline $3+(n=$ 16) respectively showed $5(31.3 \%)$ in first month, $10(62.5 \%)$ patients in second months and 1 (6.3\%) patients in third months. AFB sputum have significant correlation with time of culture conversion ( $p=0.043$; table 3 ).
AFB sputum conversion and treatment compliance Time of treatment compliance (Table 2) showed 7 (13.7\%) patients compliance in $<2$ weeks, $9(17.6 \%)$ patients in 2 weeks -1 month, 10 (19.6\%) patients in 1 month -2 months and 25 (49\%) patients $>2$ months.

The correlation between time to AFB conversion and treatment compliance (Table 4) showed patients who conversion in first month $(n=39)$ has $5(12.8 \%)$ patients with treatment

Table 3. Correlation of AFB baseline sputum on time to AFB conversion and culture conversion

\begin{tabular}{|c|c|c|c|c|c|}
\hline \multirow[t]{2}{*}{ Time (month) } & \multicolumn{4}{|c|}{ AFB Baseline } & \multirow[t]{2}{*}{$\mathrm{p}$} \\
\hline & Scanty $(n=9)$ & $1+(n=13)$ & $2+(n=13)$ & $3+(n=16)$ & \\
\hline \multicolumn{6}{|l|}{ AFB Conversion } \\
\hline $1^{\text {st }}$ & $9(100.0)$ & $9(69.2)$ & $8(61.5)$ & $13(81.3)$ & 0.528 \\
\hline $2^{\text {nd }}$ & $0(0.0)$ & $4(30.8)$ & $2(15.4)$ & $3(18.8)$ & \\
\hline $3^{\text {rd }}$ & $0(0.0)$ & $0(0.0)$ & $2(15.4)$ & $0(0.0)$ & \\
\hline $4^{\text {th }}$ & $0(0.0)$ & $0(0.0)$ & $1(7.7)$ & $0(0.0)$ & \\
\hline $5^{\text {th }}$ & $0(0.0)$ & $0(0.0)$ & $0(0.0)$ & $0(0.0)$ & \\
\hline$\geq 6^{\text {th }}$ & $0(0.0)$ & $0(0.0)$ & $0(0.0)$ & $0(0.0)$ & \\
\hline \multicolumn{6}{|c|}{ Culture conversion } \\
\hline $1^{\text {st }}$ & $7(77.8)$ & $6(46.2)$ & $4(31.3)$ & $5(31.2)$ & $0.043^{*}$ \\
\hline $2^{\text {nd }}$ & $2(22.2)$ & $6(46.2)$ & $7(53.8)$ & $10(62.5)$ & \\
\hline $3^{\text {rd }}$ & $0(0.0)$ & $1(7.7)$ & $1(7.7)$ & $1(6.3)$ & \\
\hline $4^{\text {th }}$ & $0(0.0)$ & $0(0.0)$ & $0(0.0)$ & $0(0.0)$ & \\
\hline 5 th & $0(0.0)$ & $0(0.0)$ & $1(7.7)$ & $0(0.0)$ & \\
\hline$\geq 6^{\text {th }}$ & $0(0.0)$ & $0(0.0)$ & $0(0.0)$ & $0(0.0)$ & \\
\hline
\end{tabular}

*significant 0.05

Table 4. Correlation of treatment compliance on time to AFB conversion and culture conversion

\begin{tabular}{|c|c|c|c|c|c|}
\hline \multirow[t]{2}{*}{ Time (month) } & \multicolumn{4}{|c|}{ Treatment compliance } & \multirow[t]{2}{*}{$\mathrm{p}$} \\
\hline & $\begin{array}{c}<2 \text { weeks } \\
\quad(n=7)\end{array}$ & $\begin{array}{c}2 \text { weeks - } 1 \\
\text { month }(n=9)\end{array}$ & $\begin{array}{c}1-2 \text { month } \\
(n=10)\end{array}$ & $\begin{array}{c}>2 \text { month } \\
(n=25)\end{array}$ & \\
\hline \multicolumn{6}{|c|}{ AFB Conversion } \\
\hline $1^{\text {st }}$ & $5(71.4)$ & $6(66.7)$ & $8(80.0)$ & $20(80.0)$ & 0.448 \\
\hline $2^{\text {nd }}$ & $1(14.3)$ & $2(22.2)$ & $2(20.0)$ & $4(16.0)$ & \\
\hline $3^{\text {rd }}$ & $1(14.3)$ & $0(0.0)$ & $1(11.1)$ & $0(0.0)$ & \\
\hline $4^{\text {th }}$ & $0(0.0)$ & $0(0.0)$ & $0(0.0)$ & $1(4.0)$ & \\
\hline $5^{\text {th }}$ & $0(0.0)$ & $0(0.0)$ & $0(0.0)$ & $0(0.0)$ & \\
\hline$\geq 6^{\text {th }}$ & $0(0.0)$ & $0(0.0)$ & $0(0.0)$ & $0(0.0)$ & \\
\hline \multicolumn{6}{|c|}{ Culture conversion } \\
\hline $1^{\text {st }}$ & $2(28.6)$ & $5(55.6)$ & $6(60.0)$ & $9(36.0)$ & 0.448 \\
\hline $2^{\text {nd }}$ & $4(57.1)$ & $3(33.3)$ & $4(40.0)$ & $14(56.0)$ & \\
\hline $3^{\text {rd }}$ & $1(14.3)$ & $1(11.1)$ & $0(0.0)$ & $1(4.0)$ & \\
\hline $4^{\text {th }}$ & $0(0.0)$ & $0(0.0)$ & $0(0.0)$ & $0(0.0)$ & \\
\hline $5^{\text {th }}$ & $0(0.0)$ & $0(0.0)$ & $0(0.0)$ & $1(4.0)$ & \\
\hline$\geq 6^{\text {th }}$ & $0(0.0)$ & $0(0.0)$ & $0(0.0)$ & $0(0.0)$ & \\
\hline
\end{tabular}


compliance in $<2$ weeks, $6(15.4 \%)$ in 2 weeks -1 month, $8(20.5 \%)$ in $1-2$ months and 20 (51.3\%) patients in $>2$ months. Time to AFB conversion in second months $(n=9)$ has $1(11.1 \%)$ patients with treatment compliance in $<2$ weeks, $2(22.2 \%)$ in 2 weeks - 1 month, 2 (22.2\%) in $1-2$ months and $4(44.4 \%)$ in $>2$ months. Time to AFB conversion in third months $(n=2)$ has $1(50 \%)$ patients with treatment compliance in $<2$ weeks and 1 (50\%). Time to AFB conversion in fourth months $(n=1)$ has $1(100 \%)$ patients with treatment compliance in $>2$ months. The treatment compliance was not associated with time of AFB sputum conversion ( $p$ $=0.448$ ).

\section{Culture conversion and treatment compliance}

Time to culture conversion with treatment compliance correlation showed patients who conversion in first month $(n=22)$ has $2(9.1 \%)$ patients with treatment compliance in $<2$ weeks, $5(22.7 \%)$ in 2 weeks -1 month, $6(27.3 \%)$ in $1-2$ months and $9(40.9 \%)$ patients in $>2$ months. Time to AFB conversion in second months $(n=25)$ has $4(16 \%)$ patients with treatment compliance in $<2$ weeks, 3 (12\%) in 2 weeks - 1 month, 4 (16\%) in 1-2 months and 14 (56\%) in >2 months. Time to AFB conversion in third months $(n=3)$ has $1(33.3 \%)$ patients with treatment compliance in $<2$ weeks, $1(33.3 \%)$ in 2 weeks -1 month and 1 (33.3\%) patients in $>2$ months. Time to AFB conversion in fifth months $(n=1)$ has $1(100 \%)$ patients with treatment compliance in $>2$ months (Tabel 4 ). The treatment compliance was not associated with time of culture conversion $(p=0.734)$.

\section{DISCUSSIONS}

WHO released new guidelines in May 2016, for the management of DR-TB with STR regimen of 9-12 months to update the previous guidelines in 2011, an intensive treatment phase duration of 8 months and 20 months of total treatment. STR regimen that can be given to certain DR-TB conditions or cases in order to reduce treatment failure. Monitoring the progress of DR-TB treatment with AFB sputum and culture serial each months to determine the sequential conversion of AFB baseline to the next month twice until it becomes negative ${ }^{2-4}$. The prolonged conversion has been associated with infectiousness and important for clinical management, therapeutic planning and related to treatment outcomes ${ }^{2,5,6}$.

In this retrospective study, we assessed and associated positivity of AFB baseline sputum and time to conversion with DR-TB (RR/MDR-TB) patients who received STR regimen in hospital. The present study revealed mean time of AFB sputum conversion was about one month. This result is in accordance with the studies conducted in Lianyungang City, China that have reported the median time sputum conversion was one month $(87.0 \%)$ and $(13.0 \%)$ did not convert ${ }^{10}$. Other studies in Northwest Ethiopia have reported (89.2\%) sputum conversion within 54 median days and (10.8\%) no conversion sputum smear to negative ${ }^{6}$.

Our study also revealed that for patients with positivity AFB baseline $3+$ experiencing sputum conversion in the first month. There was no significant relation between positivity of AFB baseline and time to sputum conversion. Tuberculosis infected patient might experience less productive cough after the therapy regimen and this might influence the sputum conversion result since the specimen quality might not be as good as the baseline specimen. The higher positivity AFB baseline usually related with the delay of sputum conversion but on the contrary, our research showed that the majority of patient with $3+$ baseline converted in the first month. This finding is parallel with a study done in Morroco that showed the presence of numerous bacilli on initial pretreatment sputum smears as an independent predictor of a delay of conversion of positive sputum smears ${ }^{11}$. Other factors including age, smoking habit, and HIV status might contribute to this result.

The pathogenesis of the tuberculosis infection might also contribute to the insignificantly relation between the AFB sputum conversion since the "close" foci / granuloma might not releasing a significant amount of AFB that can be detected by microscopy examination. Meanwhile, the mean time of AFB baseline sputum with time to culture conversion in this study was about two months and patients with positivity AFB baseline $3+$, also experiencing culture conversion faster than other AFB baselines. AFB sputum has significant correlation with time of culture conversion. 
Culture conversion is significantly related with the AFB baseline since the culture method is more sensitive than the AFB smear and is using a rich media that is specialized to support the growth of viable Mycobacteria. After the therapy regimen, the number of viable Mycobacteria is significantly decrease and might result in negative culture conversion. The mean time of culture conversion was comparable to the studies conducted in Nigeria that achieved sputum culture conversion by two months $(95 \% \mathrm{Cl}=1.82,230)^{12}$. However, other studies in Northwest Ethiopia have reported relatively higher culture conversion by the third $(61.7 \%)$ and sixth $(88.6 \%)$ months with median time of 72 days ( $85.5 \%)$ and (14.5\%) did not have culture conversion ${ }^{6}$. Patient with High smear grade is unlikely to convert after the first month of treatment and because of this unlikely event of sputum culture conversion, there is no need to repeat bacteriological testing after the first month of therapy ${ }^{13}$. This study also shown in our research, the majority of patient with sputum smear $2+$ and $3+$ experiencing culture conversion at their second month of treatment. The higher the smear grade, the more likely they have the culture conversion after the first month.

The late conversion of culture result in high smear grade patient might indicate dead bacilli and did not reflect treatment failure in patients with documented drug-susceptible pulmonary TB. Sputum and culture conversion at two months had a higher odds of success compared to those with no conversion at two months. Between two and four months after treatment commencement for sputum smear conversion and between four and six months are the optimum time points to predict a future successful treatment outcome ${ }^{14}$. Delay conversion prolongs the period of infectiousness and predicts higher replace and failure rates. High sputum grade were risk factor affecting prolonged the time of sputum and culture conversion ${ }^{15,16}$.

Studies in China reported smear grade $>1+(62.6 \%)$ had lower of sputum smear conversion compared with smear grade $<1+(36.7 \%)$, patients who had a higher colony count (high sputum grade) take a longer time for sputum and culture conversion ${ }^{15}$. This differs from our study that showed positivity AFB baseline 3+ experiencing sputum and culture conversion faster than other AFB baselines, this can be caused by factors associated with conversion. The important factor associated time to sputum and culture conversion is poor compliance with treatment ${ }^{17,18}$. In our study, we analyzed treatment compliance with sputum and culture conversion, mean time for treatment compliance showed more than two months and there was not associated with time of AFB sputum and culture conversion. Majority of studies showed factor that can contribute was socio-demographic ${ }^{19}$, study in China reported male patients and patients were age more than 50 years had highest incidence than younger patients ${ }^{18}$.

This was comparable to our study, from total of 51 patients a majority of patients had mean age was 51 years old. DR-TB patients were predominantly $30(58.8 \%)$ males and 21 (41.2\%) females. Lingshuang et al revealed that younger patients were more educated and regular in taking drugs with lower incidences of treatment incompliance as compared to older patients ${ }^{18}$. Pre-existing comorbidities among older patients (i.e. Diabetes Mellitus) and decreased in immunity (i.e. HIV) could be associated with risk reduced treatment compliance, loss to follow-up and increases the risk of delay conversion ${ }^{19,17,20}$. Smoking habit is also associated with the slower conversion from positive sputum smear and culture results after initiation of treatment. It is associated with increased risk of advanced and more severe disease in the form of lung cavitations, positive sputum smear and culture results ${ }^{16,8}$. Several other studies also reported risk factors affecting conversion were previous medical history of pulmonary TB, and habit (smoker), high risk behavior ${ }^{19-21}$.

\section{CONCLUSIONS}

AFB sputum has significant correlation with time of culture conversion but there was no significant relation between positivity of AFB baseline and time to sputum conversion. The treatment compliance was not associated with time of AFB sputum conversion and culture conversion. The factors may contribute affecting time to conversion was poor compliance with treatment, socio-demographic (i.e: male, age more than 50 years, history of pulmonary TB, smoker and comorbidities) potential to be associated with risk reduced treatment compliance, loss to followup and increases the risk of delay conversion. 
Further research hopefully can be examined in factors that may affect the conversion.

\section{ACKNOWLEDGMENTS}

We would like to express our sincere thanks to the Indonesia Tuberculosis International Meeting (INATIME) event which facilitated us to present this research on 5-7 April 2019 at Surabaya, Indonesia.

\section{CONFLICT OF INTEREST}

The authors declare that there is no conflict of interest.

\section{AUTHORS' CONTRIBUTION}

TK designed the experiment, concepts, drafted the manuscript, analyzed data, statistical analyzed, and designed the Fig.s and tables. ANC search literature, data collection, concepts, design, and drafted the manuscript. NPN supervised, reviewed, and edited the manuscript. $S$ reviewed the manuscript and concept experiment.

\section{FUNDING}

None.

\section{DATA AVAILABILITY}

The dataset used and/or analyzed during the current study are available from corresponding author on reasonable request.

\section{ETHICS STATEMENT}

All procedures performed in studies involving human participants were in accordance with the ethical standards of the Ethics Committee in Dr. Soetomo General Academic Hospital, Surabaya, Indonesia (1491 / KEPK / IX / 2019).

\section{REFERENCES}

1. Organization WH. Global tuberculosis report 2018. Geneva: World Health Organization; 2018. Licence: CC BY-NC-SA 3.0 IGO. WHO/CDS/TB/2018.20. Available from: http://apps.who.int/iris/bitstream

2. Waworuntu W, Subuh HM, Andriansyah, et al. Petunjuk Teknis Pengobatan TB Resisten Obat dengan Paduan Jangka Pendek di Fasyankes TB Resistan Obat. Kementerian Kesehatan Republik Indonesia, Jakarta. 2017.

3. Lange C, Chesov D, Heyckendorf J, Leung CC, Udwadia Z, Dheda K. Drug-resistant tuberculosis: An update on disease burden, diagnosis and treatment. Respirology. 2018;23(7):656-673. doi:10.1111/resp.13304
4. Bowman DC, Davies DHG, Wey DE. New drug therapies for the management of drug-resistant tuberculosis. Br J Hosp Med. 2018;79(6):C90-C92. doi:10.12968/ hmed.2018.79.6.C90

5. Chang K-C, Nuermberger E, Sotgiu G, Leung C-C. New drugs and regimens for tuberculosis. Respirology. 2018;23(11):978-990. doi:10.1111/resp.13345

6. Shibabaw A, Gelaw B, Wang S-H, Tessema B. Time to sputum smear and culture conversions in multidrug resistant tuberculosis at University of Gondar Hospital, Northwest Ethiopia. PLoS ONE. 2018;13(6):e0198080. doi:10.1371/journal.pone.0198080

7. Van Deun A, Tahseen S, Affolabi D, et al. Sputum smear microscopy in the Xpert ${ }^{\circledR}$ MTB/RIF era. Int J Tuberc Lung Dis. 2019;23(1):12-18 doi: 10.5588/ijtld.18.0553

8. Kaplan G, Post FA, Moreira AL, et al. Mycobacterium tuberculosis growth at the cavity surface: a microenvironment with failed immunity. Infect Immun. 2003;71(12):7099-7108. doi:10.1128/iai.71.12.70997108.2003

9. Ait-Khaled N, Enarson DA, Initiative ST. Tuberculosis: a manual for medical students. World Health Organization. 2003.

10. Lv L, Li T, Xu K, et al. Sputum bacteriology conversion and treatment outcome of patients with multidrugresistant tuberculosis. Infect Drug Resist. 2018;11:147154. doi:10.2147/IDR.S153499

11. Bouti K, Aharmim M, Marc K, et al. Factors influencing sputum conversion among smearpositive pulmonary tuberculosis patients in Morocco. ISRN Pulmonology. 2013;2013 doi: $10.1155 / 2013 / 486507$

12. Ige OM, Oladokun RE. Time to sputum culture conversion and treatment outcome among the first cohort of multidrug resistant tuberculosis patients in a high burden country. Indian J Tuberc. 2018;65(4):322328. doi: 10.1016/j.ijtb.2018.07.006

13. Musteikiene G, Miliauskas S, Zaveckiene J, Zemaitis M, Vitkauskiene $A$. Factors associated with sputum culture conversion in patients with pulmonary tuberculosis. Medicina. 2017;53(6):386-393. doi: 10.1016/j. medici.2018.01.005

14. Alene KA, Viney $\mathrm{K}, \mathrm{Yi} \mathrm{H}$, et al. Comparison of the validity of smear and culture conversion as a prognostic marker of treatment outcome in patients with multidrug-resistant tuberculosis. PLOS ONE. 2018;13(5):e0197880. doi:10.1371/journal. pone. 0197880

15. Diktanas S, Vasiliauskiene E, Polubenko K, et al. Factors Associated with Persistent Sputum Positivity at the End of the Second Month of Tuberculosis Treatment in Lithuania. Tuberc Respir Dis (Seoul). 2018;81(3):233240. doi:10.4046/trd.2017.0096

16. Liu Q, Lu P, Martinez L, et al. Factors affecting time to sputum culture conversion and treatment outcome of patients with multidrug-resistant tuberculosis in China. BMC Infect Dis. 2018;18(1):114. doi:10.1186/ s12879-018-3021-0

17. Mulu W, Mekonnen D, Yimer M, Admassu A, Abera B. Risk factors for multidrug resistant tuberculosis patients in Amhara National Regional State. Afr Health Sci. 2015;15(2):368-377. doi:10.4314/ahs.v15i2.9 
18. Vora SD, Gandhi RV, Vasava SA, Ganava AR, Amin G. A study of factors affecting sputum conversion in patients of pulmonary tuberculosis. National Journal of Community Medicine. 2016;7(2):142-146

19. SA MA, Salmiah M, Saliluddin S, Lim P. Factors delaying sputum conversion in smear positive pulmonary tuberculosis: a systematic review. Int J Med Sci Public Health. 2018;5(3):56-61
20. Garcell HG, Arias AV, Garcia FG, Zenon VER, Serrano RNA. Factors associated with sputum conversion in a multinational population of tuberculosis patients. Age (years). 2018;16(24):4-24.28

21. Aznar ML, Rando-Segura A, Moreno MM, et al. Prevalence and risk factors of multidrug-resistant tuberculosis in Cubal, Angola: a prospective cohort study. Int J Tuberc Lung Dis. 2019;23(1):67-72. doi:10.5588/ijtld.18.0231 